Laser Chem. 1986, Vol. 6, pp. 47-60

0278-6273/86/0601-0047\$12.00/0

(C) harwood academic publishers gmbh

Printed in Great Britain

\title{
STATE-TO-STATE COLLISIONAL DYNAMICS BY COHERENT LASER PULSE PHASE, SHAPE, AND FREQUENCY MODIFICATION
}

\author{
Mark A. BANASH and Warren S. WARREN \\ Department of Chemistry,Princeton \\ University, Princeton NJ 08544
}

Conventional coherent pulse sequences such as photon echoes measure only highly averaged relaxation rates in complex multilevel systems, such as molecules undergoing state-changing collisions. Pulse frequency, phase, and shape control lets us generate sequences which give a more detailed understanding of the dynamics. Results of dual frequency, crafted shape sequences on $I_{2}$ are presented which show that the "coherence dephasing" time $T_{2}$ is primarily due to population redistribution (energy changing collisions) in the electronically excited state, and that the electronic ground state has a much smaller cross section for such collisions. Quantitative analysis is only possible with modified laser pulse shapes which excite a single velocity component, and requires pulse sequences which correct for the hyperfine dependence of predissociation. Since this correction is rarely made in previously reported coherent transient measurements, literature values of $T_{2}$ and $T_{1}$ may not be reliable in the zero pressure limit.

\section{INTRODUCTION}

Our research efforts focus on the application of sophisticated laser or NMR pulse sequences to measure physical parameters which would be inaccessible by other means. Our recent theoretical [1] and experimental $[2,3]$ work on radiofrequency and laser pulse shape modification shows the power of this approach: pulse shapes which uniformly and completely excite a small fraction of a large inhomogeneous profile, or which excite a wide band with a narrow hole in the middle, have a variety of uses ranging from solvent 
suppression in biological NMR to spectral diffusion measurements.

One problem which is particularly well suited to our methods is the determination of detailed state-to-state dynamics in molecular collisions. A perfect two-level system has only two unique relaxation parameters: a population lifetime (usually measured by fluorescence) and a coherence lifetime (measured by photon echoes). But the real dynamics of even a very simple molecule such as $I_{2}$ is much more complex. The excited state and ground state lifetimes are certainly different at low pressure; collisional cross-sections should be state-dependent (an electronically excited state is usually "larger" than a ground state); and long range intermolecular interactions can change velocities or eliminate molecular coherence.

Dual frequency coherent transient experiments tell us not only how long the molecules stay as they were prepared, but also how they evolve toward thermal equilibrium[4,5]. They can measure population or polarization evolution, depending on the relative phases of the pulses, and give some understanding of the nature of the dominant intermolecular interactions. In our previous work $[4,5]$ we demonstrated that such sequences can selectively detect molecules which are initially excited at one particular velocity, and then migrate through collisions to a second (experimentally adjustable) velocity. Here we show that laser pulse shaping is crucial for a quantitative understanding of velocity changing dynamics.

\section{INCOHERENT DUAL FREQUENCY (IDF) EXPERIMENTS}

The basic underlying principle for our experiments is illustrated in Figures 1-2. The simplest sequence we use is two $\pi$ pulses, with a variable frequency difference. The first $\pi$ pulse (frequency $\omega_{A}$ ) excites a small fraction $\Delta \omega$ of the equilibrium Doppler distribution (Figure 1a), and leaves a similarly shaped "hole" in the ground electronic state (Figure 1b). Hard collisions during the delay $\tau$ can change electronic, vibrational or rotational states, and will deplete the initially excited population or uniformly refill the ground state hole. Weaker collisions which merely change velocities will broaden the initial distribution. Collisions which change phase but cause no energy transfer will not perturb any populations, hence they have no effect. All of these 


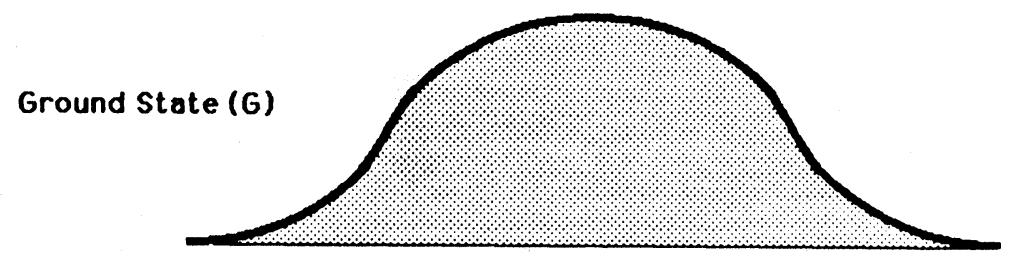

\section{Excited State (E)}

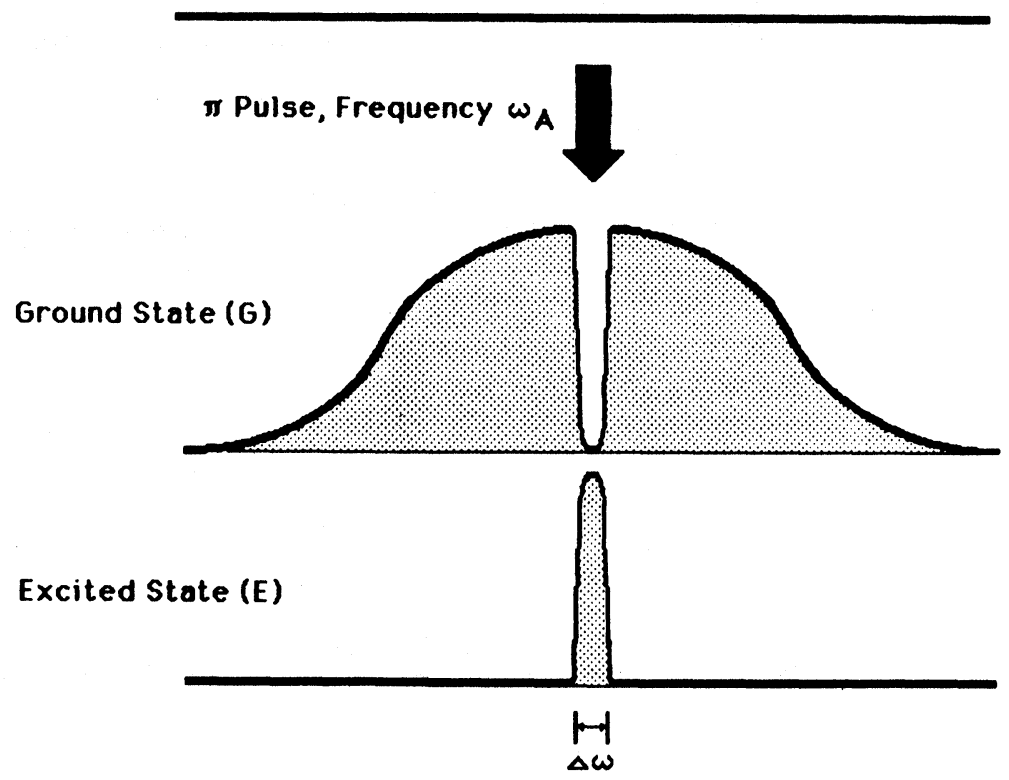

Figure 1. Excited (E) and ground (G) state velocity profiles during our incoherent dual frequency

(IDF) experiments. 


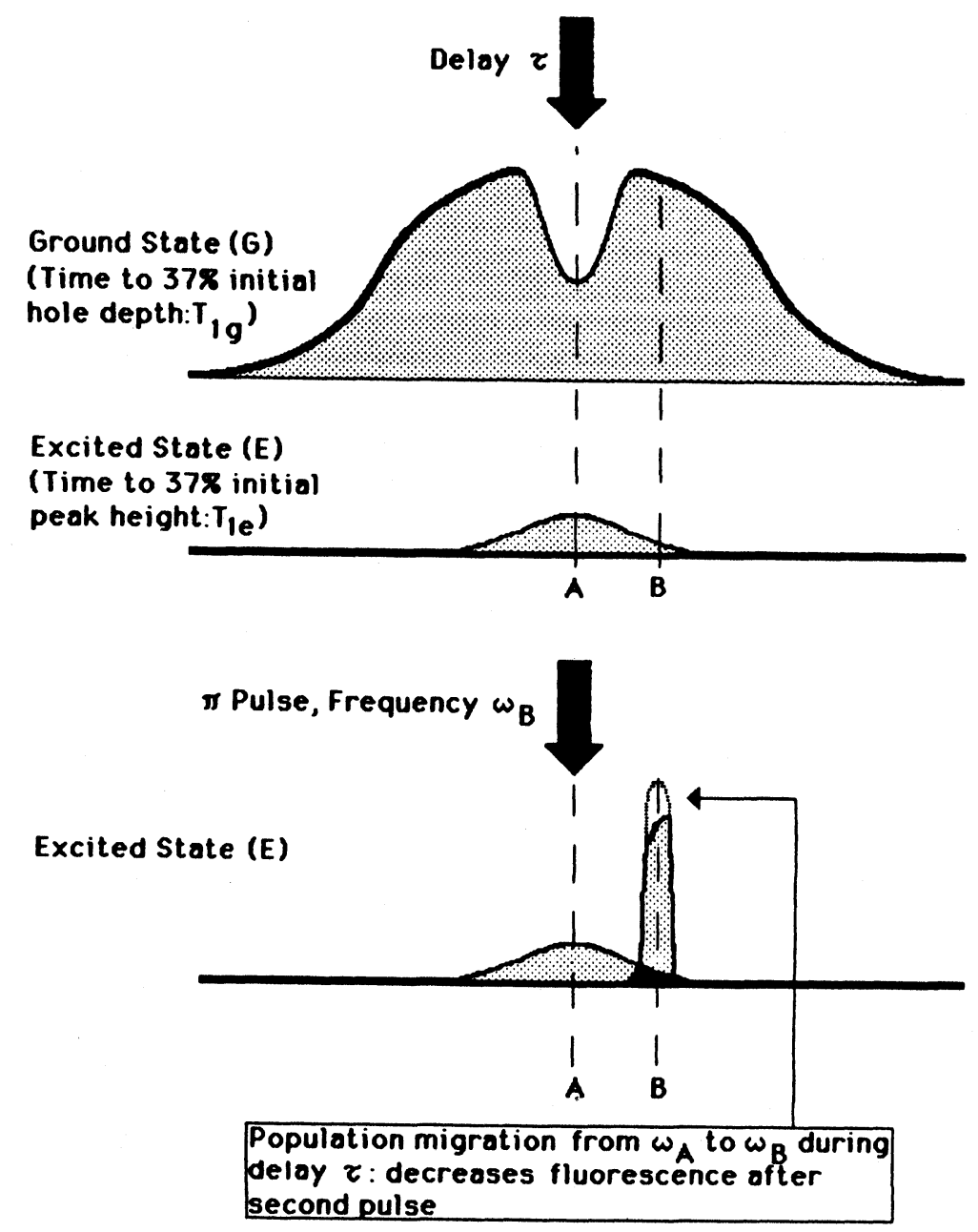

Figure 2. 
mechanisms may have different probabilities for electronically excited or ground states, so the two population distributions evolve independently.

The second $\pi$ pulse (frequency $\omega_{\mathrm{B}}$ ) deexcites any population which was initially excited by the pulse at frequency $\omega_{A}$, but which migrates during the delay $\tau$ by $\omega_{B}-\omega_{A}$ (Figure 2 ). Thus a difference spectrum (fluorescence after this sequence, minus the fluorescence induced by each pulse individually) gives the number of molecules which changed velocity by precisely the right amount to compensate for the frequency difference. Varying $\tau$ and $\omega_{A^{-}}-\omega_{B}$ gives the complete population evolution of both excited and ground states. If $\omega_{A}=\omega_{B}$ the sequence measures how long the initially excited distribution persists without evolving; the difference spectrum is a biexponential, with time constants $T_{1 g}$ and $T_{1 e}$ (see Figure $\left.2 a\right)$. If $\omega_{A}$ $\omega_{B}>\Delta \omega$ the difference spectrum is zero when $\tau=0$ (no population has migrated) or when $\tau=\infty$ (the population distribution has returned to equilibrium before the second pulse), and is negative for intermediate values of $\tau$ as shown in Figure $2 b$.

The description above makes no mention of molecular coherence, which would in fact be induced by any pulse with a flip angle unequal to $N \pi$. We excite transitions with linearly polarized radiation, which gives rotational selection rules of $\Delta F=0, \pm 1$ (the two spin-5/2 nuclei give pronounced hyperfine structure under the Doppler profile) and $\Delta M=0$. The flip angle $\mu \cdot E / h$ is proportional to $\left(F^{2}-M^{2}\right)^{1 / 2}$, by analogy with microwave spectroscopy [6]. The experiments we discuss here were done at $16,958.5 \mathrm{~cm}^{-1}$, which corresponds to $(1,58) \rightarrow(12,59)$. Such high $\mathrm{J}$ states have $\Delta \mathrm{F}=\Delta \mathrm{J}$ as a good approximate selection rule. All the $M$ states are degenerate, so our pulses in fact excite an ensemble of different transitions, and give a different flip angle for each one. This point is crucial in understanding any coherent transient data in $\mathrm{I}_{2}$, as we shall show momentarily, but has generally been ignored.

This simplified description also leaves out one important complication. Each pulse should excite a uniform and sharp velocity distribution. This means the pulse must be fairly long ( $\Delta v \Delta t \approx 1$ by the uncertainty principle). By 
this argument $10 \mathrm{Mhz}$ resolution will require transform limited, $100 \mathrm{nsec}$ pulses. The easiest way to do this is by chopping a uniform piece out of a continuous laser beam, using an acousto-optic $[7,8]$ or electro-optic modulator [9]. Such rectangular pulses have one major advantage: their effects are exactly calculable independent of electric field strength or resonance offset [10]. However they do not excite a uniform distribution. In Figure $3 a$ we show the autocorrelation function of the excited population distribution from rectangular pulse excitation. The long frequency tails imply that the IDF signal will not vanish for $\tau=0$ even for widely separated $\omega_{A}$ and $\omega_{B}$, since the pumped and probed distributions will overlap. They also imply that the signal for $\omega_{A}=\omega_{B}$ will be distorted, since molecules which change velocity through collisions will still be partly affected by the second pulse.

\section{EFFECTS OF PULSE SHAPING}

Arbitrary pulse shapes can be understood by linear response theory if the pulses are sufficiently weak, but our experiments require large population changes to have a significant signal. We wanted to understand arbitrary pulse shapes with large $(\pi / 2$ or $\pi$ ) flip angles. The fractional number of molecules which change velocity by any specified amount (say, $10 \pm 2$ meters/sec) will always be relatively small, and the sensitivity is dramatically decreased if the two pulses only transfer a small fraction of the population between excited and ground states. We have published [1] a perturbation expansion (modified from coherent averaging theory) which converges rapidly for all values of resonance offset for simple pulse shapes, and for all pulse shapes far from resonance, even when the effects are grossly nonlinear. This lets us design pulse shapes which are optimized for a uniform, complete, and localized excitation.

Only our results for a symmetric, single phase pulse will be given here. Assume the pulse extends from $t=0$ to $t=+T$. Define:

$\rho_{i n}(T / 2)=\exp \left(-i \Delta \omega I_{z} T / 2\right) \rho(0) \exp \left(+i \Delta \omega I_{Z} T / 2\right)$ 
$\rho_{f i n}(T / 2)=\exp \left(+i \Delta \omega I_{z} T / 2\right) \rho(T) \exp \left(-i \Delta \omega I_{Z} T^{T / 2}\right)$

where $\rho_{\text {in }}(T / 2)$ is the density matrix which would have been observed at $t=T / 2$ if the pulse had in fact been omitted, and $\rho_{\text {fin }}(T / 2)$ is the final prepared density matrix translated back in time to $t=T / 2$. Then

$$
\begin{aligned}
& \rho_{f i n}(T / 2)=e^{i \bar{A}} \rho_{i n}(T / 2) e^{-i \bar{A}} \\
& \bar{A}=\bar{A}^{(0)}+\bar{A}^{(1)}+\bar{A}^{(2)}+\ldots \\
& \bar{A}^{(0)}=I_{x}\left\{\int_{t=0}^{t=T} \omega_{1}(t) \cos (\Delta \omega t) d t\right\}: \text { Fourier } \\
& \text { transform of pulse } \\
& \bar{A}^{(1)}=-1 / 2 I_{z} \int_{\tau=0}^{\tau=T}\left[\int_{t=0}^{t=T} \omega_{1}(t) \omega_{1}(t+\tau) d t\right] \sin (\Delta \omega \tau) d \tau \\
& =-1 / 2 I_{z} \int_{\tau=0}^{\tau=T} G(\tau) \sin (\Delta \omega \tau) d \tau: \text { Sine Fourier } \\
& \text { transform of pulse autocorrelation function }
\end{aligned}
$$

$\bar{A}^{(2)}$ and higher order terms can usually be ignored. The equations make the pulse look as if it acted "instantaneously" at $t=T / 2$. This concept is useful because pulse length is often ill-defined (for example, a pulse shaped like a Gaussian never formally has zero amplitude, so it must be truncated at some point).

Excitation near resonance for a $\pi$ pulse is highly nonlinear. However, far from resonance only $\bar{A}(0)$ matters, so some improvements in localization are possible by just removing the high Fourier components of the pulse. The shapes we generally use in nuclear magnetic resonance are based on simple 


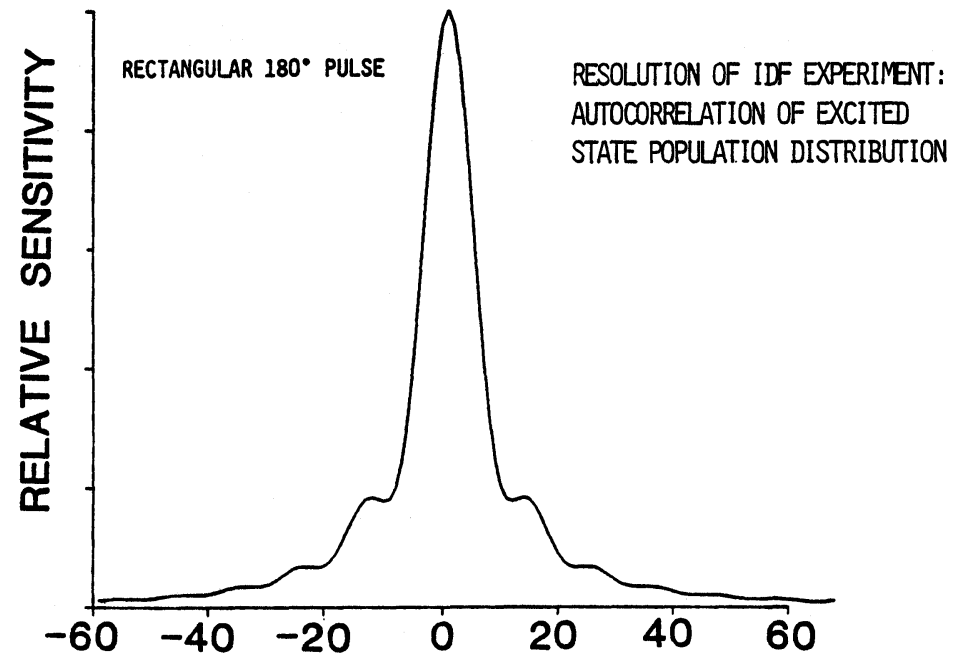

RESONANCE OFFSET(MHz,100 nsec pulse length)

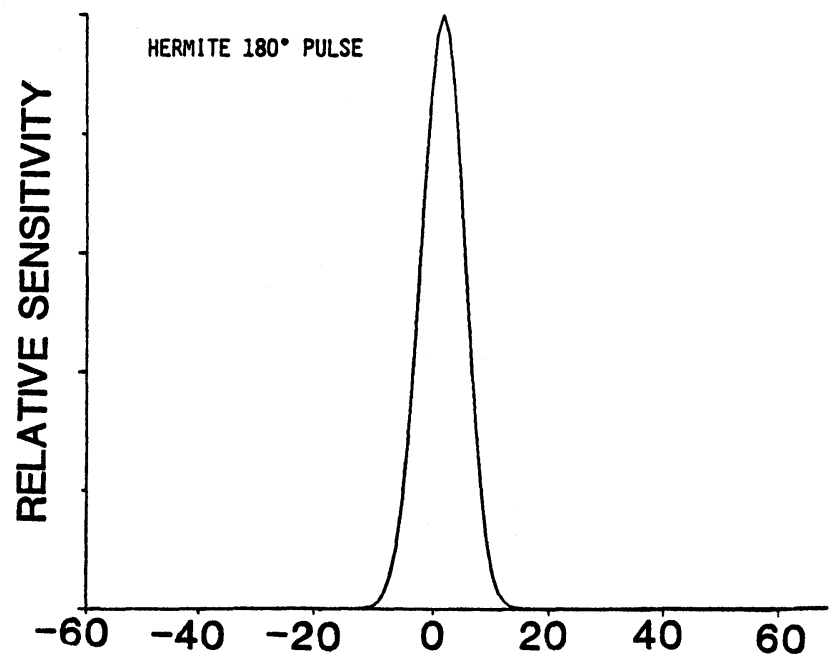

Figure 3. The frequency resolution of IDF experiments with rectangular (part a) or Hermite (part b) pulses. 
quadratic equations multiplied by a Gaussian; we call these Hermite pulses $[1,2]$.

We rely on acousto-optic modulation to generate our sequences from a ring laser beam; as we have shown in other work, the laser output replicates the phase and frequency of the radiofrequency input, and has a closely related shape. A computer controls the frequency difference by adjusting the voltage controlled oscillator in our radiofrequency driver. Pulse shaping is done at the radiofrequency level ; we can either filter the r.f. to give only a small range of frequencies, or we can generate a digital approximation to an arbitrary waveform (7 nsec resolution, 16 point approximation). Figures $3 \mathrm{a}$ and $3 \mathrm{~b}$ compare distributions which are accessible with our current equipment. Figure $3 b$ uses Hermite pulse excitation to give nearly ideal velocity resolution (an ideal, uniform population distribution would give a triangular autocorrelation), and is important for resolving fine structure of large velocity changes [1]. The distortions for $\omega_{A}=\omega_{B}$ are less severe, and so risetime modification suffices in this case; $15 \mathrm{nsec}$ rise and fall times on a $100 \mathrm{nsec}$ pulse significantly attenuates the tail of the autocorrelation.

Computerized data acquisition, delay scanning, and frequency scanning are necessary for noise reduction. The fluorescence after a two-pulse sequence is typically acquired at $40 \mathrm{KHz}$, with 1000:1 signal-to-noise ratios after one second per value of $\tau$ and $\omega_{A}-\omega_{B}$. Of course the difference spectra are less clean.

\section{RESULTS}

Some typical IDF spectra are shown in Figures 4-5. The individual spectra in this figure were taken with risetime modified pulses, and were deconvoluted to remove any residual overlap. We studied these spectra over the pressure range from 7 to $31 \mathrm{mtorr}$; in all cases the $\Delta \omega=0$ data is now a single exponential, with a decay which is qualitatively different from the fluorescence decay. A single exponential decay for $\Delta \omega=0$ has only two possible causes: either $T_{1 \mathrm{e}} \approx T_{1 \mathrm{~g}}$ or $T_{1 \mathrm{~g}}$ is extremely long. The latter case is in fact what we observe; the fluorescence difference has not returned to zero after the $5 \mu \mathrm{sec}$ maximum delay in the Figures. If $T_{1 e} \approx T_{1 g}$ then the photon echo decay must be faster 
$I_{2}$ vapor

31 mtorr

electronic transition $X\left(1 \Sigma_{0}\right) \longrightarrow B\left(3 \Pi_{0}\right)(16,958 \mathrm{~cm}-1)$

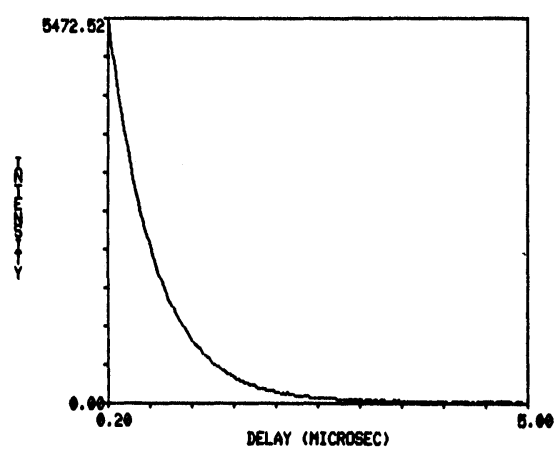

$\sqrt{\omega_{a}} \tau$

decay: 530 nsec

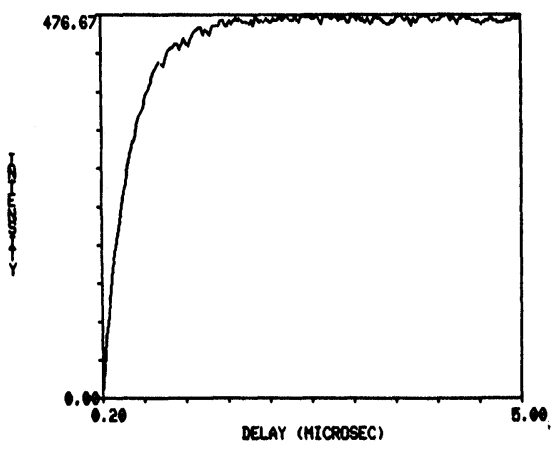

$\sqrt{\omega_{a}} \frac{\tau}{\tau \omega_{b}} L$
$\sqrt{\omega_{a}} \frac{\tau}{\tau}$

$\Delta \omega=0 \mathrm{MHz}$

decay: 322 nsec

Figure 4. IDF data for $\omega_{A}=\omega_{B}$ 


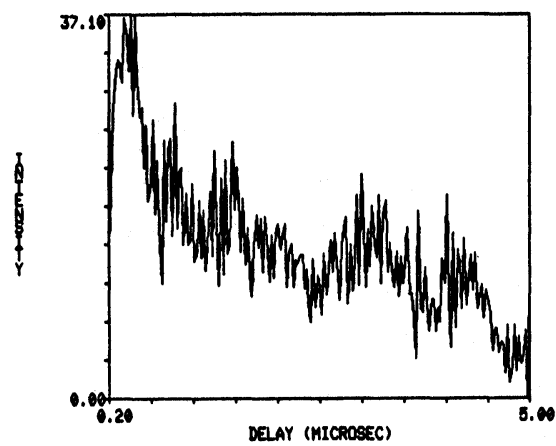

$31 \mathrm{mtorr}$

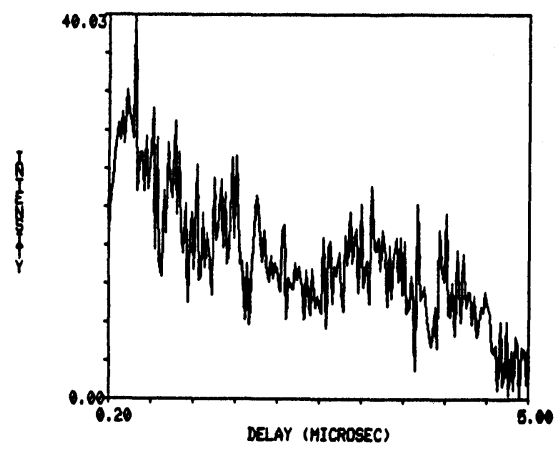

$\Delta \omega=10 \mathrm{MHz}$

$$
\Delta \boldsymbol{\omega}=20 \mathrm{MHz}
$$

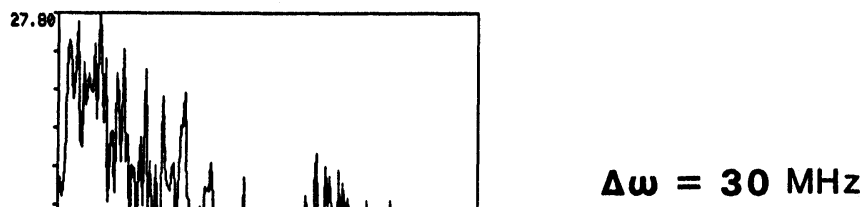

Figure 5. IDF data for $\omega_{A} \neq \omega_{B}$ 
than the IDF decay, but this is inconsistent with earlier observations by photon echoes [11]. The collisional cross-section for the $T_{1 e}$ decay is 2.2 times larger than the cross-section for fluorescence decay. Comparison with reported values of $T_{2}[11]$, which are significantly longer than $T_{1 e}$ throughout our pressure range, leads us to conclude that excited state collisions which change $F, J, v$, or velocity are causing most of the observed photon echo decay; phase interrupting collisions or collisions which repopulate the ground state hole must play only a secondary role. A more quantitative determination requires detailed $\mathrm{T}_{2}$ measurements for this particular transition, which is probably not the same as the one observed in ref. 11; such measurements are currently in progress.

Much to our initial surprise, we discovered that the zero-pressure limit for $T_{1 e}$ was systematically shorter than the zero-pressure fluorescence lifetime. This does not affect our conclusions about pressure dependence, but it does seem counterintuitive; in the absense of collisions, the initially excited velocity component should decay only by predissociation and spontaneous emission. We checked for instrumental errors (molecules wandering out of the beam between the two pulses, laser frequency jitter) but all such errors would also shorten $T_{1 g}$ measurably. In fact this observation is probably due to hyperfine coupling and predissociation of the $B$ state. The predissociation rates for the various electronically excited levels of $I_{2}$ are strongly dependent on $F$, even when $v$ and $J$ are held constant [12]; high $F$ states predissociate much faster. The $v^{\prime}=12$ level we are pumping has slow but measurable predissociation contributions to its lifetime. Furthermore, the excited distribution is strongly biased toward small values of $|M|$, because of the Rabi frequency dependence noted earlier;continuum absorption, the electronic angular momentum of the excited state, and the large spin-orbit interaction make it extremely difficult to quantify the dipole moment strengths for the various excited transitions. Yet even small variations (for example, an $\mathrm{F}$ dependence) become very important for multiple pulse sequences. Since we did not intentionally clip the wings of the tranverse (Gaussian) profile of the laser beam, most of the molecules see a relatively small flip angle (this is the reason why simple risetime modification, which merely alters the Fourier transform, gives increased resolution here). 
The relative sensitivity to different $M$ states in our experiments is proportional to the square of the population inversion after the first pulse, which is roughly proportional in this limit to the fourth power of the Rabi frequency. Thus, a three-pulse echo, our IDF experiments, and a fluorescence measurement all excited very different distributions of $M, F$, and $\mathrm{I}$.

It is possible to correct for these effects by generating pulses which simultaneously compensate for variations in the Rabi frequency and resonance offset. We have generated such pulses by starting with our Hermite shapes and adding phase shifts [13].

The data for $\Delta \omega \neq 0$ in Figure 5 show that some of the excited state population eventually reappears in adjacent velocity components. At this pressure the mean lifetime before a collision which causes $T_{1 e}$ decay (but not fluorescence loss) is roughly 1 microsecond; by that time only about $13 \%$ of the molecules are still excited. We see this population as dips which gradually become smaller as $\Delta \omega$ increases, and which peak around $1 \mu \mathrm{sec}$. There is also a gradually descending baseline, corresponding to slow refilling of the ground state hole. Out to $5 \mu \mathrm{sec}$ the fractional refilling is still very small, so it is not seen in the $\Delta \omega=0$ data (note the scale difference between these figures).

\section{CONCLUSIONS}

We have shown that dual frequency pulse sequences with crafted laser pulse shapes can give a detailed picture of collisional dynamics. Hyperfine splittings and predissociation in $\mathrm{l}_{2}$ distort comparisons of measurements involving different numbers of pulses (such as fluorescence decays and photon echoes) for most of the excited vibronic states. We can verify, however, that the process which causes loss of polarization in photon echo measurements is not a "T $T_{2}$ process" in the usual sense; it has to do with population redistribution in the excited state, not loss of phase coherence per se. Measurements of relaxation times with phase and amplitude modulated pulses are needed to make fully quantitative determinations of the relative importance of different mechanisms.

We wish to thank Kevin Lehmann, Dick Miles, Jack Gelfand and Bob Field for useful and stimulating discussions. This work was supported by the 
National Science Foundation under grant CHE-8405944.

References:

1. W. S. Warren, J. Chem. Phys. 815437 (1984).

2. M.McCoy and W. S. Warren, J. Mag. Reson. 65178 (1985)

3. J. Gutow, M. McCoy, F. Spano and W. S. Warren, Phys. Rev. Lett. 551090 (1985)

4. M. A. Banash, J. Gutow and W. S. Warren, J. Luminescence $31-32855$ (1984)

5. W. S. Warren and M. A. Banash, Coherence and Quantum Optics V (L. Mandel and E. Wolf, editors; Plenum, New York, 1984) p.959

6. C.H. Townes and A. L. Schawlow, Microwave Spectroscopy (Dover, New York, 1975) p.264

7. W. S. Warren and A. H. Zewail, J. Chem. Phys. 755956 (1981) ; J. Chem.

Phys. 782279 (1983)

8. T. E. Orlowski and A.H. Zewail, J. Chem. Phys. 701390 (1979)

9. R. G. Brewer and R. L. Shoemaker, Phys. Rev. A6 2001 (1972)

10. R. Feynman, F. L. Vernon and R. W. Hellwarth, J. Appl Phys. 2849 (1957)

11. E. Sleva and A. H. Zewail, Chem. Phys. Lett. 110582 (1984)

12. J. Vigue, M. Broyer and J. C. Lehmann, J. Physique 42949 (1981); L.

Brewer and J. Tellinghuisen, J. Chem. Phys. 563929 (1972)

13. M. Navratil, M. McCoy, J. Bates and W. S. Warren, J. Chem. Phys. (to be submitted) 